\title{
Long-duration Carrier-Smoothed-Code Algorithm for GPS Positioning
}

\author{
H.-S. Chen, L.-S. Wang \\ Institute of Applied Mechanics \\ National Taiwan University, Taipei, Taiwan, R.O.C. \\ F.-R. Chang \\ Department of Electrical Engineering \\ National Taiwan University, Taipei, Taiwan, R.O.C.
}

\begin{abstract}
To manage the problems arisen during long-term positioning by using the GPS (Global Positioning System) signals, the so-called long-duration carrier-smoothed-code (LCSC) algorithm was developed. Based on the classical carrier-smoothed-code (CSC) method, a Kalman filter is used to predict the carrier phase and to compensate the lost carrier phase once cycle slip happens. A fuzzy-control-based tuning algorithm is applied to adjust the weightings between the carrier signal and code signal in the CSC method. A least-square method is used to solve the inevitable problem of satellite changes during long-term navigation. From both static and dynamic experiments, the LCSC indeed provides a more stable method in long-term positioning.
\end{abstract}

\section{Introduction}

The GPS(Global Positioning System) has been extensively used in recent years for surveying, navigation, time transfer, etc., cf. [1] and the references therein. To get centi-meter-level accuracy positioning, the carrier phase observables must be adopted, along with the differential techniques. However, the use of carrier phase observables alone may require a long convergence period for initialization, and is thus subjected to serious problems caused by cycle slip, if used in real-time. On the other hand, algorithms based on code observables can provide less accurate positioning data (meters, DGPS) right after the signal is tracked. To avoid solving the problem of integer ambiguity and still get satisfactory accuracy in time, the Carrier-Smoothed-Code (CSC) algorithm was developed to synthesize the carrier phase observables and the code observables. Although the algorithm is sound, jumps in positioning may still appear when cycle slips and satellite changes occur during long-term positioning. It is desired to alleviate these jumps to yield a smoother positioning algorithm.

In this paper, the Long-duration CSC (LCSC) algorithm is developed to handle these problems. A Kalman filter is used to predict the occurrence of cycle slips and to smooth the carrier-smoothed-code pseudo-ranges. With the smoothed CSC pseudo-ranges, it is noted that it is not necessary to re-initialize the CSC method if cycle slip occurs. Instead, an algorithm based on the fuzzy-control theory is designed to adjust the weightings between the carrier phase observables and the code observables in the CSC algorithm once cycle slip happens. To handle the problem of satellite changes, a least-square scheme according to the different weightings between satellite signals is applied. Similar idea can be used to solve the problems with predictable cycle slips, such as appearing when crossing a known tall building in the city. In order to verify the proposed algorithm, both static and dynamic experiments were conducted. It was shown that the LCSC algorithm indeed provides more stable positioning data in long-term measurement.

\section{The GPS Observables}

In the Global Positioning System (GPS), there are 24 satellites moving around the Earth and transmitting navigation signals which are received by the receivers on the ground or near the Earth. Due to the long transmission channels, clock biases, and other uncertainties, measurement errors such as the satellite ephemeris errors, satellite clock biases, receiver clock biases, ionospheric delay, tropospheric refraction, multipath effect, and other random noises appear in the model for the observables. From the satellite signals, two kinds of pseudo -range observables can be obtained, i.e. the code pseudo-range and carrier-phase pseudo-range. They can be modeled as follows. From the PRN code, the pseudo-range from the satellite $j$ to the receiver $A$ can be written as 


$$
P_{A}^{j}=\rho_{A}^{j}+c\left(d t^{j}-d T_{A}\right)+\left(d_{\text {ion }}+d_{\text {trop }}+\varepsilon\right)_{A}^{j},
$$

where $\rho_{A}^{j}$ denotes the true range between satellite $j$ and receive $A, c$ is the speed of light, $d t^{J}, d T_{A}$ denote the clock biases of satellite $j$, and receive $A$, respectively, $d_{\text {ion }}, d_{\text {trop }}$ represent the ionospheric and tropospheric effect respectively, and $\varepsilon$ denotes the random noise.

On the other hand, we may count the carrier beat phase, namely, the difference between the $L I$-band carrier wave from the satellite and the reference signal generated by the receiver, and obtain the carrier-phase pseudo-range:

$$
\Phi_{A}^{j}=\rho_{A}^{j}+c\left(d \dot{p}^{j}-d T_{A}^{\prime}\right)+\lambda \cdot N_{A}^{j}+\left(-d_{i o n}+d_{\text {trop }}+\varepsilon\right)_{A}^{j}
$$

in which $\lambda$ denotes the wavelength of the $L I$ carrier, i.e. $19 \mathrm{~cm}$, and $N_{A}^{j}$ is the integer ambiguity caused by the tracking process. Although it is fixed if the signal is continuously tracked, the integer ambiguity is subject to change if cycle slip happens. From both the code pseudo-ranges and the carrier-phase pseudo-ranges, one may be able to find the position of the receiver through some numerical process. However, using code observable alone, the positioning process is almost instant, but is not very accurate (a few meters for DGPS). Using carrier-phase observables alone, the algorithm can lead to accurate position (centi-meters for KGPS), but it requires long initialization process, due to the problem associated with the integer ambiguity. Especially for real-time long-term positioning, the problems caused by cycle slip are inevitable, and the algorithms using carrier phase alone are not very reliable. In order to have reasonably accurate position within short period, the two pseudo-ranges are synthesized to yield the carrier-smoothed-code (CSC) pseudo-range as shown below.

\section{The Carrier-Smoothed-Code Method}

As discussed above, the two pseudo-ranges may be combined to obtain the CSC pseudo-range:

$$
\hat{P}(t)=z_{1}(t) P(t)+z_{2}(t) \cdot(\hat{P}(t-1)+\Phi(t)-\Phi(t-1))
$$

where $z_{1}, z_{2}$ denotes the weightings of the code and carrier-phase, respectively, and we have $z_{1}+z_{2}=1$. The weightings are set initially as $z_{1}=1, z_{2}=0$, so that the code pseudo-range dominates. As time goes by, the weighting $z_{1}$ for the code observables becomes smaller, and thus the carrier phase observables become dominate, which yields accurate positioning in steady state. The rule $z_{1}(t)=1 / t$ is typically used. Once cycle slips occur, the weightings are reset. By using the CSC pseudo-ranges, the positioning algorithm can be then performed to find the position of the receiver with reasonable accuracy in short period. Moreover, to attain the centimeter-level accuracy in steady state, the differential techniques need to be applied.

For two receivers close to each other, the single difference between the receivers can be computed as

$$
\hat{S}(j, t)=\rho_{B A}^{j}+c \cdot\left(d T_{A}-d T_{B}\right)+\varepsilon_{B A}^{j}
$$

where

$$
\begin{aligned}
& \hat{S}(j, t)=\hat{P}_{B}^{j}(t)-\hat{P}_{A}^{j}(t), \\
& \rho_{B A}^{j}=\rho_{B}^{j}-\rho_{A}^{j}, \\
& \varepsilon_{B A}^{j}=\varepsilon_{B}^{j}-\varepsilon_{A}^{j} .
\end{aligned}
$$

It is noted that the errors in (1) on ionosphere, troposphere, and the satellite clocks are eliminated. In addition, to get rid of the clock biases in the receivers, the double difference is then used

$$
\hat{D}(k, j, t)=\rho_{B A}^{k j}(t)+\varepsilon_{B A}^{k j},
$$

in which

$$
\begin{aligned}
& \hat{D}(k, j, t)=\hat{S}(k, t)-\hat{S}(j, t), \\
& \rho_{B A}^{k j}=\rho_{B A}^{k}-\rho_{B A}^{j}, \\
& \varepsilon_{B A}^{k j}=\varepsilon_{B A}^{k}-\varepsilon_{B A}^{j} .
\end{aligned}
$$

Let $\mathrm{r}_{A}=\left(x_{A}, y_{A}, z_{A}\right), \mathrm{r}_{B}=\left(x_{B}, y_{B} z_{B}\right)$ denote the position vectors of the receiver $A$ and $B$, respectively. The true range functions $\rho_{A}^{j}$ can be linearized about a reference point $C$, in the neighborhood of $A$ and $B$. By observing four satellites simultaneously, say $j, k, m, n$. and performing the linearization, equation (2) can be expanded and approximated by

$$
\left[\begin{array}{l}
\hat{D}(k, j, t) \\
\hat{D}(m, j, t) \\
\hat{D}(n, j, t)
\end{array}\right]=\left[\begin{array}{l}
\left(\rho_{C}^{\prime \prime}-\rho_{C}^{\prime \prime}\right)^{T} \\
\left(\rho_{C}^{\prime m}-\rho_{c}^{\prime \prime}\right)^{T} \\
\left(\rho_{C}^{\prime n}-\rho_{C I I}^{\prime}\right)^{T}
\end{array}\right]\left[\begin{array}{l}
x_{B}-x_{A} \\
y_{B}-y_{A} \\
z_{B}-z_{A}
\end{array}\right]+\left[\begin{array}{l}
\varepsilon_{B A}^{b} \\
\varepsilon_{B A}^{\prime m} \\
\varepsilon_{B A}^{n}
\end{array}\right]
$$

where $\rho_{c}^{\prime k}$ is the first derivative of the range function evaluated at the reference point $C$. In vector form, the above equation can be expressed as

$$
\hat{\mathbf{D}}(t)=H(t) \mathbf{b}(t)+\varepsilon(t),
$$

where $\mathbf{b}=\mathbf{r}_{B}-\mathbf{r}_{A}$ is the relative vector from $A$ to $B$, and is the unknown to be sought. In equation (3), the double difference observables are obtained from the 
measurements, and the matrix $H$ can be computed from the ephemeris data in the GPS message. The unknown vector $\mathbf{b}$ is then obtained by using the least square process.

If there are more than four satellites in vision, it may be possible to give the appropriate weightings on the various observations. Let the weighting matrix be denoted by $W$, and the goal is to minimize the cost function $\mathbf{e}^{T} W \mathbf{e}$, where $\mathbf{e}=\hat{\mathbf{D}}-H \mathbf{b}$. The least square solution can be found to be

$$
\widetilde{\mathbf{b}}=\left[H^{T} W H\right]^{-1} H^{T} W \hat{\mathbf{D}} \text {. }
$$

By appropriately choosing the weighting matrix, the problem of predictable cycle slips or satellite changes can be handled smoothly.

\section{The Long-duration Carrier-Smoothed-Code Algorithm}

For long-duration positioning using GPS, the inevitable problems caused by cycle slips and satellite changes may lead to jumps on the positioning, which is un-desirable. It is thus required to smooth the position data to increase the reliability of the algorithm. First of all, the cycle slip detection is performed. The idea of using the Kalman filter (cf. e.g. [5]) to detect the occurrence of cycle slip has been reported in [2] with the following model for the carrier phase observables:

$$
\left[\begin{array}{c}
\phi_{k+1} \\
\dot{\phi}_{k+1} \\
\ddot{\phi}_{k+1}
\end{array}\right]=\left[\begin{array}{ccc}
1 & \Delta t & 0.5(\Delta t)^{2} \\
0 & 1 & \Delta t \\
0 & 0 & 1
\end{array}\right]\left[\begin{array}{c}
\phi_{k} \\
\dot{\phi}_{k} \\
\ddot{\phi}_{k}
\end{array}\right]+\left[\begin{array}{c}
w_{1, k} \\
w_{2, k} \\
w_{3, k}
\end{array}\right],
$$

and

$$
z_{k}=\left[\begin{array}{lll}
1 & 0 & 0
\end{array}\right]\left[\begin{array}{lll}
\phi_{k} & \dot{\phi}_{k} & \ddot{\phi}_{k}
\end{array}\right]+v_{k} .
$$

By using Kalman filter on the above dynamical equations, the carrier phase may be predicted. If the difference between the predicted carrier phase and the carrier phase observable is a few cycles, the occurrence of cycle slip is then reported.

If cycle slip occurs within one second, the predicted carrier phase may be used to continue the positioning process. If the signal is totally lost for one second, a reasonable prediction for the code observable may be used as follows.

$$
\begin{aligned}
P(t)= & P(t-1)+ \\
& 0.5 \cdot[(P(t-2)-P(t-3))+(P(t-1)-P(t-2))] .
\end{aligned}
$$

This compensation for one-second interrupt can be done even if there are only four satellites under tracked. If the cycle slip occurs more than one second, and there are more than four satellites tracked, it may be possible to recover the positioning algorithm through the following process. Let cycle slip occur in the signal from satellite $m$ at epoch $t_{0}$. The position determined from the other satellites may be used to recover the lost one. In particular, at time $t_{0}$, the estimated baseline vector $\widetilde{\mathbf{b}}\left(t_{0}\right)$ is computed from the signals without having cycle slip, and the estimated single difference $\widetilde{\hat{S}}\left(m, t_{0}\right)$ for satellite $m$ is obtained from

$$
\left[\begin{array}{c}
\hat{S}\left(k, t_{0}\right)-\hat{S}\left(j, t_{0}\right) \\
\vdots \\
\tilde{\hat{S}}\left(m, t_{0}\right)-\hat{S}\left(j, t_{0}\right)
\end{array}\right]=H\left(t_{0}\right) \tilde{\mathbf{b}}\left(t_{0}\right) .
$$

Then at the epoch $t_{0}+1$, the positioning process continues with

$$
\begin{aligned}
\hat{S}\left(m, t_{0}+1\right)= & z_{1}\left(t_{0}+1\right) \cdot S_{p}\left(m, t_{0}+1\right)+ \\
& z_{2}\left(t_{0}+1\right) \cdot\left[\tilde{\hat{S}}\left(m, t_{0}\right)+S_{\Phi}\left(m, t_{0}+1\right)-S_{\Phi}\left(m, t_{0}\right)\right],
\end{aligned}
$$

where $S_{p}, S_{0}$ denote the single differences for the code and carrier-phase observables, respectively.

Despite the appearance of cycle slip, the above idea may be used to smooth the CSC pseudo-range at any time. The following process may be performed:

Step 1: Compute $\widetilde{\mathbf{b}}(t)$ at epoch $t$

Step 2: Compute the estimated double differences, and then the single differences

$$
\begin{aligned}
& \widetilde{\hat{\mathbf{D}}}=H \widetilde{\mathbf{b}}, \\
& \widetilde{\hat{S}}(k, t)=\widetilde{\hat{D}}(k, j, t)+\hat{S}(j, t) .
\end{aligned}
$$

Step 3: For each satellite, update $\hat{S}$ at epoch $t+1$ by

$$
\begin{aligned}
\hat{S}(k, t+1)= & z_{1}(t+1) \cdot S_{p}(k, t+1)+ \\
& z_{2}(t+1) \cdot\left[\hat{\hat{S}}(k, t)+S_{\odot}(k, t+1)-S_{\odot}(k, t)\right]
\end{aligned}
$$

Step 4: Continue the positioning process.

With this algorithm, the CSC single differences are smoothed, which yield smoother positioning data.

During long-term positioning, once cycle slip happens, the classical treatment is to reset the weightings to be $z_{1}=1, z_{2}=0$, However, some discontinuity of positioning may arise due to the abrupt changes of the weightings between code and phase. The algorithm then needs a new convergent process before the required accuracy being attained. However, combining with the previous smoothing method, it is not necessary to restart the process. Instead, a fuzzy controller may be used to adjust the weightings. Let the speed of the receiver and the DOP value are chosen 
as the input variables with the membership functions shown below:
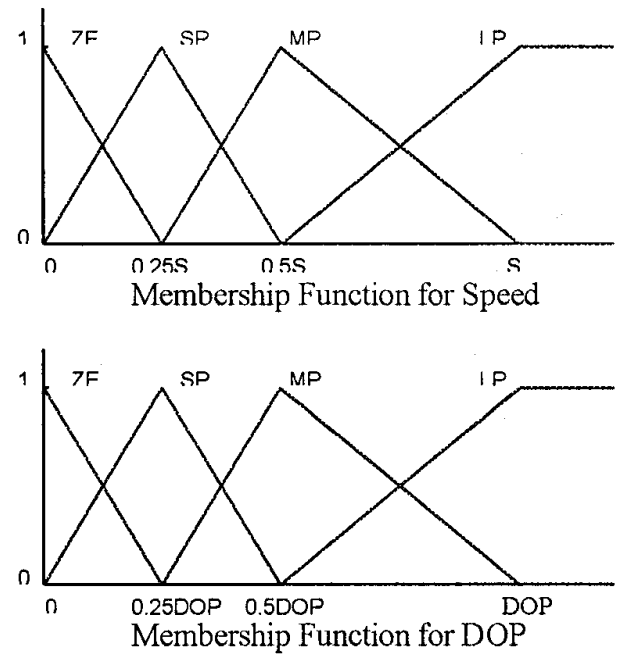

The output variable is chosen as $1 / z_{1}$, with the other weighting being determined accordingly. The membership function is selected as

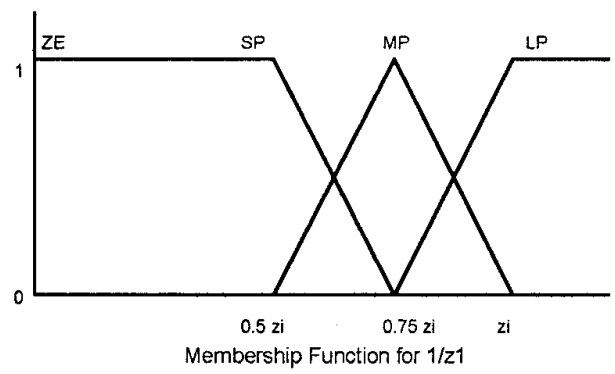

To attain smooth positioning, the value of $z i$ can not be small. Moreover, in the fuzzy control, the inference rule is set as shown in the following table:

\begin{tabular}{|c|c|c|c|c|}
\hline SpeedDOP & ZE & SP & MP & LP \\
\hline ZE & ZE & ZE & LP & LP \\
\hline SP & ZE & LP & LP & MP \\
\hline MP & LP & MP & MP & SP \\
\hline LP & MP & MP & SP & SP \\
\hline
\end{tabular}

With the information of the speed of the receiver and the DOP value, the desired weightings can be then obtained by invoking the fuzzy controller.

In addition to the above revisions on the CSC method, we may handle the problem associated with satellite change by using different weightings on the satellite signals. For predictable cycle slips, such as the set of satellites below the horizon, and the crossing of a known building, etc., the weightings of the signals which may be subjected to the problem of cycle slip should be reduced. In fact, assuming that the channels are independent, the covariance matrix of the errors can be found to be

$$
\operatorname{cov}()=\left[\begin{array}{ccc}
\left(\sigma_{A}^{j}\right)^{2}+\left(\sigma_{B}^{j}\right)^{2}+\left(\sigma_{A}^{k}\right)^{2}+\left(\sigma_{B}^{k}\right)^{2} & \left(\sigma_{A}^{\prime}\right)^{2}+\left(\sigma_{B}^{j}\right)^{2} & \\
\left(\sigma_{A}^{j}\right)^{2}+\left(\sigma_{B}^{j}\right)^{2} & \ddots & \ddots
\end{array}\right]
$$

where $\sigma_{A}^{k}$ denotes the variance of the errors for the signal received by receiver $A$ from satellite $k$. If satellite $k$ is likely to disappear, we may increase the variances $\sigma_{A}^{k}, \sigma_{B}^{k}$ in the covariance matrix. The weighting matrix may be then selected as the inverse of the covariance matrix,

$$
W=[\operatorname{cov}(\varepsilon)]^{-1}
$$

and the desired position vector is then computed by applying the formula (4) in the least-square method.

On the other hand, for satellite just rising from the horizon, the weightings for its signal may be increased according to the elevation. Similar idea may be used to solve the problem of crossing known buildings in the city.

Combining the above techniques, the LCSC algorithm can be used to smooth the positioning, which is justified by the experiments described below.

\section{The Experimental Results}

In order to assess the effectiveness of the LCSC algorithm, both static experiments and dynamical experiments are performed, with using the Motorola Oncore GPS receivers, cf. [4]. For the static experiments, the two receivers are stationarily located with a few meters apart. To check the cycle slip detection and compensation algorithm, the cycle slips were intentionally added in the observed carrier phases within 150 seconds. Figure 1 shows the performance of the one-second compensation scheme. It is observed that the accuracy of the classical CSC method becomes worse when cycle slip occurs at $50 \mathrm{sec}$, while the compensation scheme with Kalman filter works quite well. If the cycle slip extends for a few seconds, say $10 \mathrm{sec}$. in Figure 2, the cycle slip compensation scheme described in the previous section gives better result than the classical $\mathrm{CSC}$ method, as shown in Figure 2 .

The results of long-term static positioning are shown in Figure 3. The experiment was conducted continuously for twelve hours, with the last six hours 
plotted. In the Figure, cureve I represents the result by using CSC, curve II is obtained by applying the CSC pseudo-range smoothing algorithm, while the outcome of the CSCS algorithm is plotted as curve III. Obviously, the proposed LCSC algorithm is superior to the classical CSC method, and the scheme dealing with satellite change is necessary.

For real-time, dynamical experiments, the two receivers are put at both ends of a rotating shaft, respectively. The structure of the rotating mechanism is shown in Figure 4. With the beam being continuously rotated for about three hours, both CSC method and the LCSC algorithm are used to find the relative position between the two receivers. The evolution of computed lengths by both methods are depicted in Figure 5. It is seen that at about 54 minutes, there is a cycle slip occurred and the CSC method is subjected to abrupt change. However, the positioning by using LCSC algorithm is not affected significantly. Furthermore, the $x, y$ coordinates obtained by both methods are plotted in Figure 6 and Figure 7 , respectively. Significant improvement by using LCSC algorithm is observed.

\section{The Conclusions}

In order to have a stable algorithm in long-term positioning, methods dealing with cycle slips and satellite changes need to be developed. The classical $\mathrm{CSC}$ method is enhanced here by including the scheme of cycle-slip detection and compensation, the strategies of adjusting the weightings between codes and phases, and the weightings between satellites. From the experimental results, the algorithm proposed in the paper, the LCSC algorithm, indeed yields good results on reducing the discontinuities in the positioning, and shortening the convergence process. More advanced filtering methods or analysis may be used to obtain better results on smoothing the signals and on the selection of weightings.

\section{References}

[1] Kaplan, E.D., Understanding GPS: Principles and Applications, Artech House, Inc., 1996.

[2] Mertikas, S.P., C. Rizos, "On-line Detection of Abrupt Changes in the Carrier-phase Measurement of GPS," Journal of Geodesy, 1997, pp. 469-482.

[3] Jin, X.X., "A New Algorithm for Generating Carrier Adjusted Differential GPS Corrections," Journal of Geodesy, 1996.
[4] Motorola, Oncore Receiver Users ' Guide, 1994.

[5] Grewal, M.S., A.P. Andrews, Kalman Filtering Theory and Practice, Prentice-Hall, Inc, 1993.

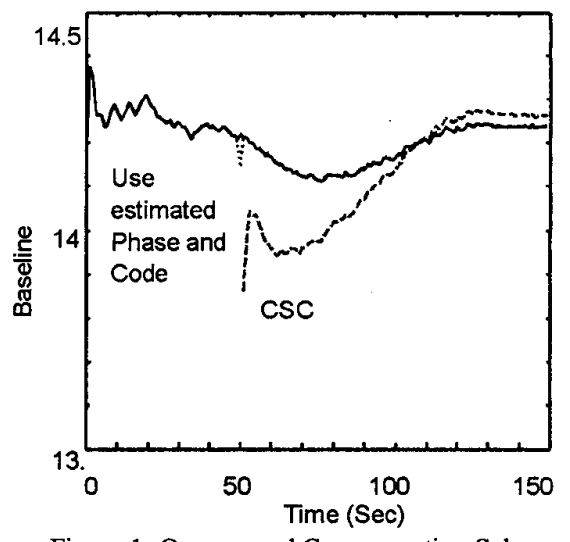

Figure 1. One-second Compensation Scheme

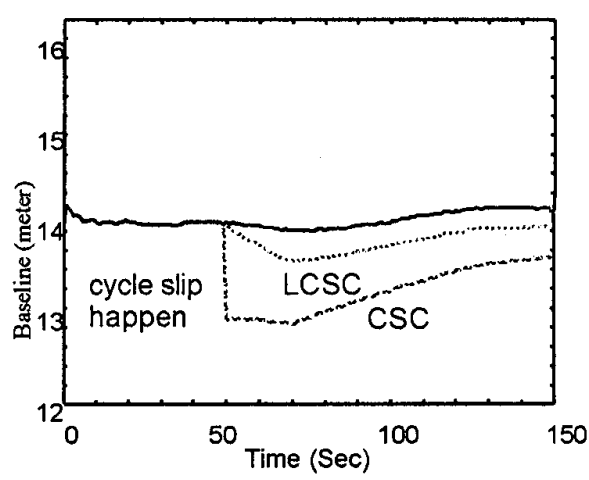

Figure 2. Cycle-slip Compensation Scheme

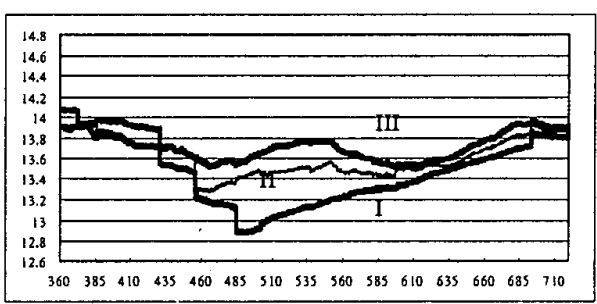

Figure 3. Baseline for Static Experiment 


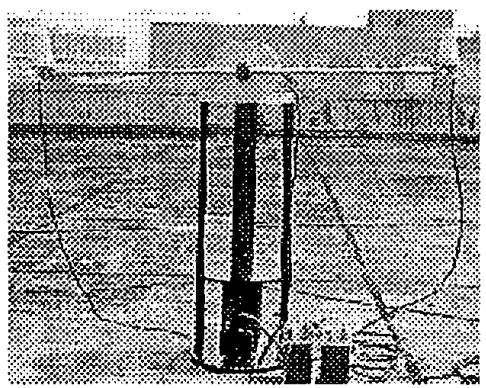

Figure 4. Rotating Mechanism

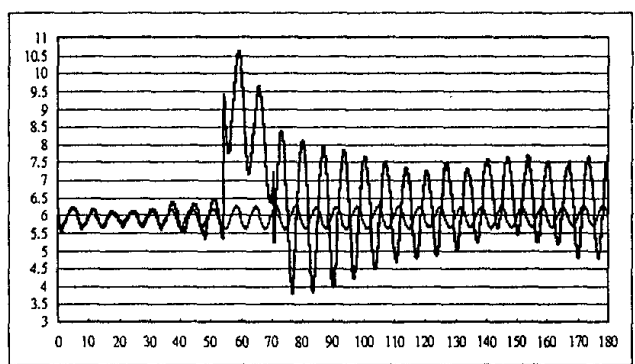

Figure 5. Baseline for Rotating Beam By CSC and LCSC

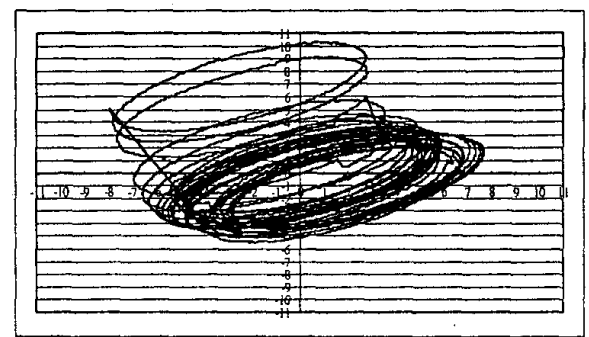

Figure 6. $x-y$ Plot for Rotating Beam By CSC

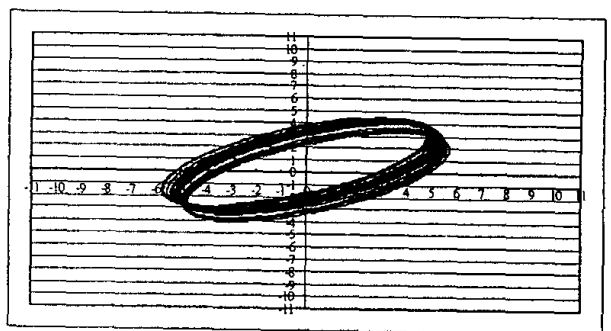

Figure 7. $x$-y Plot for Rotating Beam By LCSC 